\title{
Jin Shofu Starch Nanoparticles for the Consolidation of Modern Paintings
}

\author{
Andrea Casini, David Chelazzi,* and Rodorico Giorgi*
}

Cite This: ACS Appl. Mater. Interfaces 2021, 13, 37924-37936

Read Online

ABSTRACT: Matte, porous, and weakly bound paint layers, typically found in modern/contemporary art, represent an unsolved conservation challenge. Current conservation practice relies on synthetic or natural adhesives that can alter dramatically the optical properties of paints. Alternatively, we propose a novel nanostructured consolidant based on starch, a renewable natural polymer. We synthesized starch nanoparticles (SNPs) to boost their penetration into the porous painted layers; upon solvent evaporation, the particles were expected to adhere to the pigments thanks to their large surface area and abundant $-\mathrm{OH}$ groups. The SNPs were formulated through a bottom-up approach, where gluten-removed Jin Shofu wheat starch was gelatinized and then

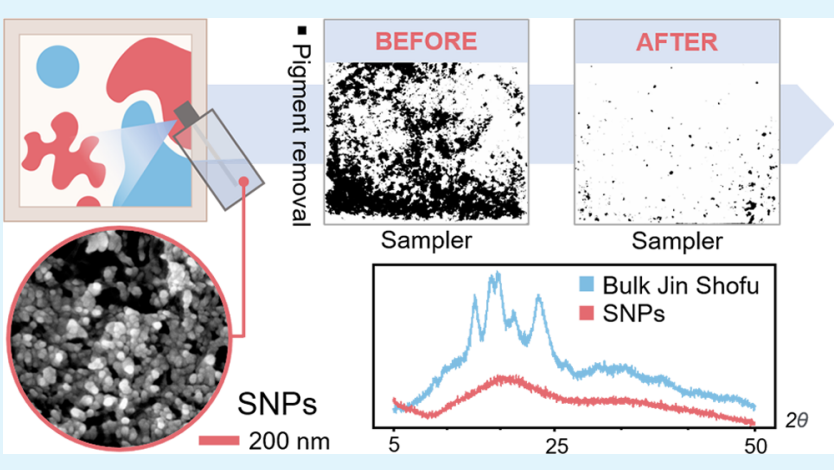
precipitated in a nonsolvent. The low gelatinization temperature of wheat starch is likely key to favor disassembly in alkali and reassembly in the nonsolvent. The synthesis conditions can be tuned to obtain amorphous SNPs of ca. $50 \mathrm{~nm}$ with acceptable polydispersity. The particles swell in water to form nanosized gel-like fractal domains (as observed with cryogenic electron microscopy), formed by the organization of smaller units in polymer-rich and -deficient regions. Aqueous and hydroalcoholic particles' dispersions were assessed on aged ultramarine blue mock-ups that mimic degraded modern/contemporary paints. The consolidation effectiveness was evaluated with a specifically designed in-house protocol: the SNPs distribute across the paint section and strongly increase pigments' cohesion while preserving the original optical properties of the painted layer, as opposed to dispersions of bulk starch that simply accumulate on the paint surface, forming superficial glossy films. The Jin Shofu SNPS proved to be a new promising tool for the consolidation of weakened paintings, opening perspectives in the formulation and application of consolidants for modern and contemporary art.

KEYWORDS: starch nanoparticles, nanoprecipitation, Cryo-TEM, modern art, paintings consolidation

\section{INTRODUCTION}

Matte, porous, and weakly bound paint layers, typically found in modern art, represent nowadays an important conservation challenge. Most modern and contemporary artists use industrial painting formulations rich in additives and not conceived to last long, which they freely altered, experimenting with mixtures; as a consequence, paintings can exhibit fragile or powdering surfaces even before natural aging takes its course. Then, exposure to environmental fluctuations and severe climatic conditions, in addition to outdoor pollution, accelerates the loss of binding media. ${ }^{1}$ This is particularly true for paints with matte surfaces, which typically have high values of "pigment volume concentration" (PVC), i.e., the binder's quantity is generally much lower than the pigment's. These painted layers exhibit poor cohesive/adhesive properties, show an opaque and powdery appearance, and have different consolidation needs than works where the concentration of the binder is higher. ${ }^{2}$ Examples include, for instance, works by Edvard Munch, which suffer from extreme deterioration as a result of artistic experimentation and, in some cases, owing to the fact that the artist stored some of his paintings outdoors. ${ }^{3}$

Common conservation practice lacks satisfactory consolidation materials to target such demanding challenges. Traditionally, restorers apply natural and synthetic organic adhesives to readhere the powdering painted layers, but these approaches prove detrimental as the adhesives can alter dramatically the optical properties of the treated surfaces. ${ }^{1,4}$ Besides, some synthetic formulations, such as those based on poly(vinyl acetate), are known to emit volatile organic compounds (VOCs) that damage either the paint layer or the canvas support. ${ }^{5}$

Received: June 14, 2021

Accepted: July 15, 2021

Published: July 28, 2021 
Alternatively, the development of tailored consolidants for art objects starting from renewable natural sources appears as a sustainable route, provided the new materials cope with specific requirements. ${ }^{6}$ Among natural materials, starch is a promising candidate, being low cost, renewable, and nontoxic. Besides, it has good compatibility with paint layers, which in some cases already contain dextrin (processed wheat or corn starch) as a filler and extender. Indeed, starch has been proposed as an adhesive in paper conservation in the form of aqueous dispersions prepared from the bulk polymer. However, we expected that its effectiveness and applicability would be boosted if starch nanoparticles (SNPs), rather than the bulk polymer, were used. First, the reduced size of SNPs should allow efficient penetration inside the porous paint layers, avoiding the formation of thick surface coatings that provide only superficial consolidation and can alter the paintings' appearance. Upon solvent evaporation, the particles are expected to form a homogeneous network through the paint section and microjunctions that strengthen the weak paint layers. Second, the high surface-to-volume ratio of the SNPs is expected to enhance the adhesivity of starch to the pigments, thanks to the great number of surface interactions via hydroxyl groups. ${ }^{8,9}$

The literature reports different synthetic paths for the formulation of starch nanoparticle dispersions, including topdown and bottom-up approaches. ${ }^{9,10}$ Acid hydrolysis is commonly adopted, as it removes the amorphous portion of the polymer, ${ }^{11}$ but this method was not suitable for our purposes due to the high volumes of concentrated acid required, the low yield of the process (below 1\%), long preparation time (ca. 1 week), and high polydispersity of the obtained particles. Besides, amorphous particles have a higher polymer chain mobility, which might help in relaxation of mechanical stresses in paint layers and favor polymer-pigment interactions. Amorphous domains also have the advantage of being more transparent since random chain entanglement tends to let light pass through, as opposed to scattering crystalline centers. ${ }^{12}$ Alternatively to acid hydrolysis, a bottomup method based on the dissolution of starch in alkali and the precipitation of the gelatinized polymer in a nonsolvent (ethanol) was deemed a promising approach ${ }^{9,13}$ being simple, robust, time-effective, and able to produce nanoparticles with the desired size. Here, we changed and optimized this method and selected "Jin Shofu" (an easily accessible gluten-removed and purified wheat starch) as the starting bulk material. This highly purified starch has been used in Japan for over 1000 years and is still employed by local paper conservators owing to its capacity to form adhesive layers that are more flexible than those obtained with rice starch, making Jin Shofu the material of choice for book conservation. Its use in the form of SNPs, however, is completely unprecedented and so is its application to consolidate paint layers. Wheat starch is an optimal choice for the bottom-up SNPs synthesis since its gelatinization temperature is lower than that of other common starches (corn, rice, potato), which facilitates the alkali disassembly of the polymer chains and, thus, their reassembly into nanoparticles when exposed to a nonsolvent.

The main precipitation parameters that influence the final particles' shapes and dimensions were optimized, and the most promising SNPs dispersions, in terms of stability and particle characteristics, were selected for consolidation tests on aged painting mock-ups that mimic the degraded and poorly bound paint layers typically found in modern/contemporary art. Paint consolidation was assessed using an in-house testing system based on microsampling, coupled with macro- and microphotography, and two-dimensional (2D) Fourier transform infrared (FTIR) imaging. The optical properties of the treated surfaces were also assessed by colorimetry.

\section{EXPERIMENTAL SECTION}

Materials. The Jin Shofu wheat starch paste used for this study is produced by Paper Nao (Tokyo, Japan). Ethanol absolute anhydrous (EtOH, purity $\geq 99.9 \%$, Carlo Erba), ethanol absolute denaturated (denEtOH, purity $\geq 99.2 \%$, Carlo Erba), and potassium hydroxide (KOH, $\geq 86 \%$, for analysis, pellets, Fluka) were used as purchased. Ultramarine blue pigment was supplied by Zecchi (Florence, Italy). Water was purified by a Millipore MilliRO-6 Milli-Q gradient system (resistivity $>18 \mathrm{M} \Omega \mathrm{cm}$ ).

Starch Nanoparticles (SNPs) Synthesis. Starch nanoparticles were obtained through alkali treatment and nonsolvent precipitation. Starch suspensions $(0.6,0.8,1 \% \mathrm{w} / \mathrm{v})$ were prepared by mixing 0.06 , 0.08 , and $0.1 \mathrm{~g}$ of starch powder with $10 \mathrm{~mL}$ of $\mathrm{KOH}$ aqueous solution $(0.1 \mathrm{M})$. The mixtures were heated at $80{ }^{\circ} \mathrm{C}$ under constant stirring for $30 \mathrm{~min}$ in a two-neck round-bottom flask equipped with a condenser to avoid moisture evaporation, obtaining slightly opalescent and homogenous starch dispersions. The dispersions were quickly cooled to room temperature and added dropwise into EtOH to obtain the SNPs, varying the starch dispersion/nonsolvent ratios (1:10 and 1:40), under constant stirring rate $(1300 \mathrm{rpm})$. The SNPs dispersions were kept for another $30 \mathrm{~min}$ at room temperature under the same stirring conditions and then centrifuged $(8500 \mathrm{~g}$ at 8 ${ }^{\circ} \mathrm{C}$ for $5 \mathrm{~min}$ ), discarding the supernatant, and rinsing the SNPs with denEtOH to remove excessive water and $\mathrm{KOH}$. The yield of the synthetic process (formation of SNPs) was calculated simply by weighing the dried particles after precipitation in $\mathrm{EtOH}$ and $\mathrm{KOH}$ removal. Subsequently, the nanoparticles were freeze-dried in vacuum conditions overnight and then ground and stored in a desiccator at 25 ${ }^{\circ} \mathrm{C}$, before redispersion. SNPs dispersions of 1 and $5 \mathrm{~g} / \mathrm{L}$ were obtained redispersing separately the ground powder into ultrapure water and different water-ethanol blends, stirring the dispersions overnight at $1300 \mathrm{rpm}$. The dispersions were then dialyzed for $24 \mathrm{~h}$ using a high-retention seamless cellulose membrane $(23 \mathrm{~mm} \varnothing$, molecular weight cutoff $12400 \mathrm{Da}$, Sigma-Aldrich) to adjust the final $\mathrm{pH}$ to $\sim 7$, and then treated by ultrasonication at $8{ }^{\circ} \mathrm{C}$, using a Branson 450 digital sonifier equipped with a microtip $(5 \mathrm{~mm} \varnothing)$.

Physicochemical Characterization of the Dried SNPs. The morphological analysis of SNPs was performed with a field emission gun scanning electron microscope SIGMA (FEG-SEM, Carl Zeiss Microscopy $\mathrm{GmbH}$, Germany), using an acceleration potential of 3 $\mathrm{kV}$ and a $3.9 \mathrm{~mm}$ working distance. Dried SNPs, obtained by varying the starch initial concentration $(0.6,0.8$, and $1 \% \mathrm{w} / \mathrm{v})$ and solvent/ nonsolvent ratios (1:10 and 1.40), were analyzed. The particles' size distributions were then obtained by ImageJ software, averaging on 30 measurements, and reported as normal distributions. ${ }^{14}$

Differential scanning calorimetry (DSC) was used to study the thermal behavior of the dried SNPs and bulk Jin Shofu starch. Dry powders $(6-7 \mathrm{mg})$ were placed inside hermetically sealed steel pans, heated from 25 to $180^{\circ} \mathrm{C}$, and then cooled down at the rate of 10 ${ }^{\circ} \mathrm{C} / \mathrm{min}$ under a nitrogen stream two times. For each specimen, three independent measurements were carried out.

X-ray diffractometry (XRD) on Jin Shofu bulk starch and SNPs was carried out using a Bruker New D8 Da Vinci diffractometer (radiation at $\mathrm{Cu} \mathrm{K} \alpha_{1}=1.54056 \AA, 40 \mathrm{kV} \times 40 \mathrm{~mA}$ ), equipped with a Bruker LYNXEYE-XE detector and a secondary monochromator. Data were collected in the $2 \theta$ range of $5-50^{\circ}$, with a slide of $0.6 \mathrm{~mm}, 2.5^{\circ}$ soller slit configuration, step size of $0.02^{\circ}$, and time per step of $57.2 \mathrm{~s}$.

Physicochemical Characterization of SNPs Dispersions. The hydrodynamic radius $\left(R_{\mathrm{h}}\right)$ and electrokinetic potential ( $\zeta$ potential) of the SNPs dispersions were measured on 1 and $5 \mathrm{~g} / \mathrm{L}$ water and water-ethanol $\left(\mathrm{H}_{2} \mathrm{O}: \mathrm{EtOH}\right.$ 85:15 v/v) formulations, right after the redispersion process. 
Dynamic light scattering (DLS) measurements were carried out using a Brookhaven instrument equipped with a 400-channel digital autocorrelator (BI 9000AT), a Nd:YAG laser $(\lambda=532 \mathrm{~nm}, 20 \mathrm{~mW})$, and a temperature-controlled sample cell holder. A $400 \mu \mathrm{m}$ pinhole aperture was employed for the EMI 9863B/350 photomultiplier tube, and Decalin (decahydronaphthalene) was used as the refractive index matching fluid to reduce stray light. Band-pass filters were used to modulate the scattering light intensity of the most opalescent dispersions $(5 \mathrm{~g} / \mathrm{L})$. To obtain the particle size distributions from the measured autocorrelation functions, the CONTIN algorithm was used, and the results were reported as number and intensity plots (see the SI). Each specimen was analyzed at a constant temperature of 25 ${ }^{\circ} \mathrm{C}$, and three independent measurements were taken.

The $\zeta$ potentials of the SNPs dispersions were analyzed by means of a Zeta Plus, Brookhaven Instruments Corporation, Holtsville, NY. The electrokinetic potential values were obtained according to the Helmholtz-Smoluchowski equation considering the electrophoretic mobility $u$ :

$$
\zeta=\frac{\eta}{\varepsilon} u
$$

where $\eta$ is the medium viscosity and $\varepsilon$ is the medium dielectric permittivity. ${ }^{15}$ The $\zeta$-potential values were obtained as averages of three separate measurements for each SNPs dispersion.

Cryoe-transmission electron microscopy (Cryo-TEM) was used to characterize the nanometric structure of water-swollen SNPs. An aliquot $(\sim 3 \mu \mathrm{L})$ of each $5 \mathrm{~g} / \mathrm{L}$ SNPs dispersion (water and waterethanol) was deposited on a glow-discharged $\mathrm{Cu}$ carbon-coated grid. The liquid in excess was blotted away with filter paper using a ThermoScientific Vitrobot, and the samples were instantly frozen by rapid immersion in liquid ethane. The samples were thus observed using a ThermoScientific "Glacios" microscope with an X-FEG highbrightness gun of $200 \mathrm{keV}$.

The stability of the SNPs dispersions in water and in waterethanol blends was evaluated by monitoring their settling velocity through UV-vis spectroscopy. Sedimentation kinetics of water and water-ethanol SNPs dispersions $(5 \mathrm{~g} / \mathrm{L})$ was monitored using a Cary UV-vis 3500 spectrophotometer (Agilent Technologies) at a fixed wavelength $(500 \mathrm{~nm})$ : decreases in the absorbance were ascribed to aggregate formation and sedimentation. Baseline corrections were performed before sample analysis, employing distilled deionized water and water-ethanol blends. A first stability screening of different SNPs water-ethanol dispersions (ethanol content from 0 to $50 \% \mathrm{v} / \mathrm{v}$ ) was carried out over $30 \mathrm{~min}$ acquiring UV-vis spectra every $3 \mathrm{~s}$ at $25^{\circ} \mathrm{C}$. Later, the sedimentation kinetics of $5 \mathrm{~g} / \mathrm{L}$ water and $\mathrm{H}_{2} \mathrm{O}: \mathrm{EtOH}$ 85:15 (v/v) dispersions were followed over 1 month: spectra were collected every $24 \mathrm{~h}$ for the first 5 days, and then once per day after 5 , $10,15,20$, and 25 days. Three independent measurements were performed on each sample.

Preparation of Painting Mock-Ups. Matte painting mock-ups with low pigment cohesion and high pigment volume concentration (PVC) were prepared by mixing ultramarine blue powder and deionized water $(1: 75, \mathrm{w} / \mathrm{w})$ under constant stirring. The obtained paint paste was applied by a spatula directly on a glass slide with no binder to simulate poor cohesion and adhesion onto the support. The freshly painted mock-ups were dried in an oven at $60{ }^{\circ} \mathrm{C}(20 \% \mathrm{RH})$ overnight and then wetted with deionized water and kept at $-20{ }^{\circ} \mathrm{C}$ for $2 \mathrm{~h}$. The frozen mock-ups were then thawed at $60^{\circ} \mathrm{C}(20 \% \mathrm{RH})$ for $2 \mathrm{~h}$, and the wet freeze-thaw cycle was repeated six times, which produced highly powdery painted surfaces.

Characterization of the Treated Paint Mock-Ups. Macrophotography and microphotography were used to characterize the visual appearance of the mock-ups before and after treatment with the SNPs. The aged mock-ups were treated using either bulk starch dispersions or dispersions of SNPs. In the first case, bulk Jin Shofu starch (water dispersion, $5 \mathrm{~g} / \mathrm{L}$ ) was gelatinized by heating at $98{ }^{\circ} \mathrm{C}$ for ca. $1.5 \mathrm{~h}$ and allowed to cool down to room temperature.

Vis-light macrophotography was carried out using a Canon EOS 5D Mark II Full Frame DSLR camera equipped with a Sigma $105 \mathrm{~mm}$ f/2.8 EX macro lens, with a light setup of $45^{\circ}$ on both sides for reducing shadow formation on the surface of the samples. To study possible color alterations induced by the treatment, aged mock-ups $\left(2.5 \times 5 \mathrm{~cm}^{2}, 1 \mathrm{~mm}\right.$ thick $)$ were treated by spraying either bulk starch or SNPs dispersions $\left(5 \mathrm{~g} / \mathrm{L}\right.$, in water and $\mathrm{H}_{2} \mathrm{O}: \mathrm{EtOH}$ 85:15 v/v). Color changes were assessed after one or three spraying rounds $(1 \mathrm{~mL}$ each). Colorimetry measurements were performed relying on the CIE-Lab* color system. The color difference $\Delta E^{*}$ between samples was calculated as

$$
\Delta E^{*}=\left[\left(\Delta L^{*}\right)^{2}+\left(\Delta a^{*}\right)^{2}+\left(\Delta b^{*}\right)^{2}\right]^{1 / 2}
$$

where $\Delta L^{*}$ is the difference in luminosity, $\Delta a^{*}$ is the difference in the green-red scale, and $\Delta b^{*}$ is the difference in the blue-yellow scale. ${ }^{16}$ Macrophotography was coupled with an X-rite ColorChecker Passport, as a reference. Custom camera profiles based on camera/ lens/lighting combinations were thus created via dedicated X-rite software (ColorChecker Camera Calibration) and then processed by the Adobe Photoshop Lightroom plug-in (which provides standardized lighting conditions). The CIE-Lab coordinates were collected using the color sampler tool integrated in Adobe Photoshop. For each sample, the coordinates were recorded as an average of 10 independent measurements.

Visible-light microphotographs were collected in the reflectance mode by means of a Reichert Zetopan optical microscope, equipped with EPI $5.5(20 \times)$ and $11(40 \times)$ objectives, and coupled with a Nikon Digital Sight DS-Fi2 camera.

FTIR 2D imaging was used to characterize the distribution of starch on the surface and through the cross section of the ultramarine blue paint mock-ups treated with bulk starch or SNPs dispersions (5 $\mathrm{g} / \mathrm{L}$, in water and $\left.\mathrm{H}_{2} \mathrm{O}: \mathrm{EtOH} 85: 15 \mathrm{v} / \mathrm{v}\right)$. Mock-ups of $2.5 \times 5 \mathrm{~cm}^{2}(1$ $\mathrm{mm}$ thick) were treated by three spray applications $(1 \mathrm{~mL}$ each). FTIR imaging was carried out using a Cary 620-670 FTIR microscope (Agilent Technologies), equipped with a focal plane array (FPA) $128 \times 128$ detector. This setup was selected as it allows discriminating compounds with different chemical compositions on a surface, down to a spatial resolution of a few microns. ${ }^{17}$ The detection limit of the FPA detector was recently shown to be on the order of picograms per pixel. $^{18}$ The spectra were recorded directly on the surface of the samples in reflectance mode, with open aperture and a spectral resolution of $8 \mathrm{~cm}^{-1}$, acquiring 128 scans for each spectrum. A "single tile" map has dimensions of $700 \times 700 \mu \mathrm{m}^{2}$ (corresponding to $128 \times 128$ pixels) and a pixel size of $5.5 \mu \mathrm{m}$ (i.e., each pixel has dimensions of $5.5 \times 5.5 \mu \mathrm{m}^{2}$ and is related to an independent spectrum). In each $2 \mathrm{D}$ map, the intensity of characteristic bands of starch was imaged. The chromatic scale of the maps shows increasing absorbance of the bands as follows: blue $<$ red $<$ orange $<$ yellow.

To assess the consolidation effect of the SNPs, an in-house consolidation assessment protocol was used, where the treated and untreated paint mock-up surfaces were sampled in a controlled and reproducible way, and then, the amount of pigment removed by sampling was measured semiquantitatively. Paint mock-ups of $2.5 \times 5$ $\mathrm{cm}^{2}$ (1 mm thick) were treated by a single spray application $(1 \mathrm{~mL})$ of either bulk starch or SNPs dispersions $(5 \mathrm{~g} / \mathrm{L}$, in water and $\mathrm{H}_{2} \mathrm{O}: \mathrm{EtOH} 85: 15 \mathrm{v} / \mathrm{v}$ ). White fiberglass filters (GF3 grade, $47 \mathrm{~mm} \varnothing$ circles, chm by CHMLAB Group) were cut into $1 \mathrm{~cm}^{2}$ squares and glued with a double-layer tape to a Teflon support of the same size, which was in turn glued to the bottom of a glass vial filled with sand, for an overall weight of $100 \mathrm{~g}$. The obtained sampler was placed (no pressure applied) onto the ultramarine blue mock-up surface for $30 \mathrm{~s}$. Sampling was repeated 3 times in different surface areas to account for inhomogeneity in superficial cohesion of the pigments. After sampling, the filters were detached from the glass vial, placed on a glass slide, and analyzed through vis-light macrophotography using the same setup as described before. Macrophotographs of the pigment-stained filters were thus processed with the GNU Image Manipulation Program (GIMP) to obtain a photoplane analyzable with ImageJ software. ${ }^{19}$ RGB channels were then split; the image of each channel was binarized (using an automatically set average threshold) and compared to the original 8-bit grayscale image using a cross-correlation analysis plug-in developed by Chiga et al. ${ }^{20}$ The 


\section{STARCH SOLUTION:ETHANOL RATIO}

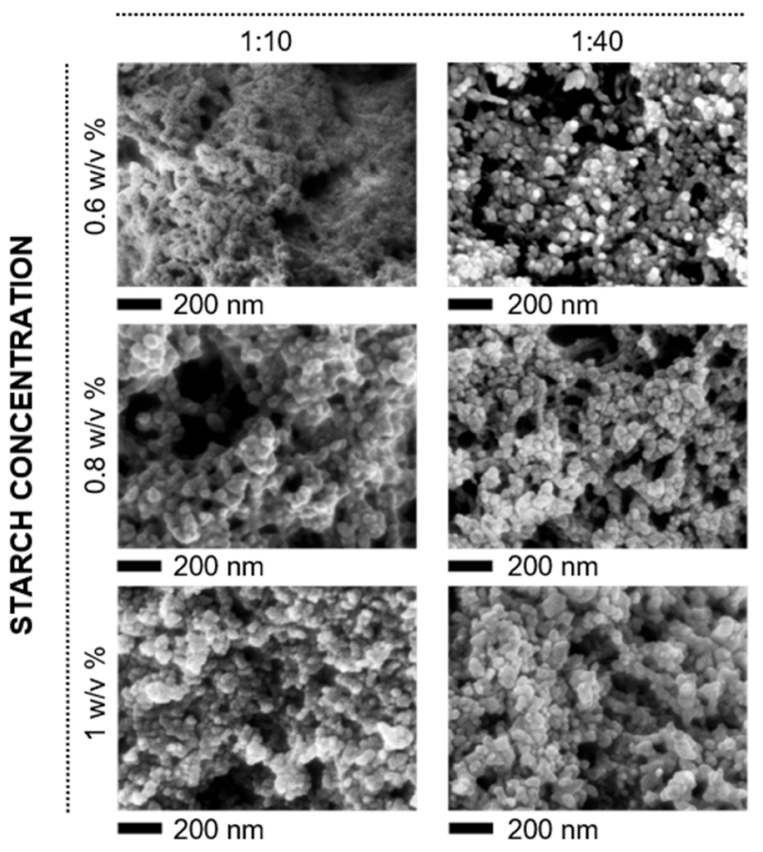

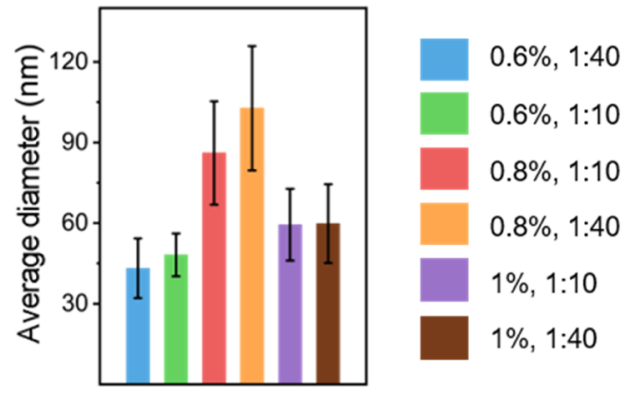

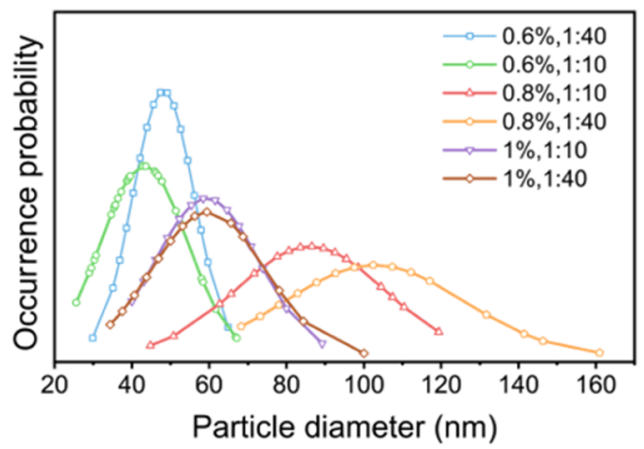

Figure 1. (Left) SEM images of Jin Shofu freeze-dried SNPs obtained by alkali treatment and nonsolvent precipitation, using starch concentrations of $0.6,0.8$, and $1.0 \%(\mathrm{w} / \mathrm{v})$ and water/ethanol ratios of $1: 10$ and $1: 40(\mathrm{w} / \mathrm{w})$, respectively. (Right) Particles' average sizes and size distributions, as obtained from the SEM images.

algorithm estimates an average statistics for each local region with a predefined size (72 pixels), on two aligned images ("reference" and "target"). The average basis weight of the entire assessed area is given by $\bar{z}$, and the local basis weight of the local subarea is given by $z\left(m_{\mathrm{i}}\right.$, $\left.n_{j}\right):$

$$
z=\left(\frac{1}{m n} \sum_{i=1}^{m} \sum_{j=1}^{n} z\left(m_{i}, n_{j}\right)\right)
$$

where $m$ and $n$ are the numbers of local regions along the $X$ and $Y$ directions, respectively, and $\left(m_{i}, n_{j}\right)$ corresponds to the local subarea position. The Pearson correlation coefficient $R$, and thus its squared value $\left(R^{2}\right)$, is estimated according to

$$
R=\frac{\sum_{i=1}^{m} \sum_{j=1}^{n}\left(f\left(m_{i}, n_{j}\right)-\bar{f}\right)\left(g\left(m_{i}, n_{j}\right)-\bar{g}\right)}{(m n-1) \sigma_{f} \sigma_{g}}
$$

where $f$ and $g$ are the means of the average statistics of the "reference" and "target" images, respectively, and $\sigma_{f}$ and $\sigma_{g}$ are their standard deviations. ${ }^{20}$

The channel image with the best correlation $(R)$ and determination $\left(R^{2}\right)$ coefficients was considered as the most representative, and its original, nonbinarized 8-bit version was used for subsequent elaborations. In our case, for the ultramarine-blue-stained filter images, the red channel was selected. The original red channel photoplane was then binarized, this time using separately each of the 17 threshold mathematical functions provided by ImageJ. The 17 resulting images were correlated with the original 8-bit red channel image, and only the threshold functions that produced $|R|>0.95$ and $R^{2}>0.9$ were kept, while the others were discarded. The percentage of black pixels was measured and averaged over the selected images, and the obtained value was directly used as an expression of the pigment relative coverage (\%) on the filter surface.

\section{RESULTS AND DISCUSSION}

Under ambient conditions, starch is relatively insoluble in water and many common solvents, but aqueous solutions can be obtained using elevated pressure and/or temperature and high $\mathrm{pH}$. Too harsh conditions are usually not considered to avoid changes in the molecular weight of the polymer, while too mild conditions may not be sufficient for complete dissolution. ${ }^{21}$ Dimethyl sulfoxide (DMSO) has been adopted by some authors and coupled with the use of autoclaves, but the usage of this solvent was discouraged in our case, as DMSO has low volatility and good solving power toward several paint components; therefore, possible solvent residues in the final SNPs formulation could be transferred to treated paint layers and prove harmful. The removal of DMSO is commonly a lengthy process that would make the synthetic path time-consuming.

Bulk starch can be dissolved in alkalis, where the majority of the polymer hydroxyl groups are ionized, and the crystal structure of starch chains can be destroyed. Overall, alkali dissolution followed by precipitation in a nonsolvent is a feasible and robust synthetic process to obtain SNPs and does not use harmful compounds that could be found in the final product. Alkali residues in the starch particles can be easily removed by dialysis of the dispersion overnight (see Experimental Section). For the precipitation, ethanol was selected over methanol (a better nonsolvent) to reduce the toxicity of the process.

According to the literature, for the synthesis of SNPs, starch dispersions $(1 \% \mathrm{w} / \mathrm{v})$ are treated with either $\mathrm{NaOH}$ or $\mathrm{KOH}$, at alkali concentrations mostly of $1 \mathrm{M}$, varying the dissolution time (e.g., from $30 \mathrm{~min}$ to $1.5 \mathrm{~h}$ ). Direct heating, autoclaves, and microwave ovens are typically used to provide thermal energy. In our case, we observed that dissolution with $0.1 \mathrm{M}$ $\mathrm{KOH}$ at $80{ }^{\circ} \mathrm{C}$ under stirring for $30 \mathrm{~min}$ produced a homogeneous opalescent starch dispersion with no evident solid residue. It must be noticed that the possibility of using low amounts of alkali in the starch disassembly step makes the overall process "greener" and less expensive. Besides, 

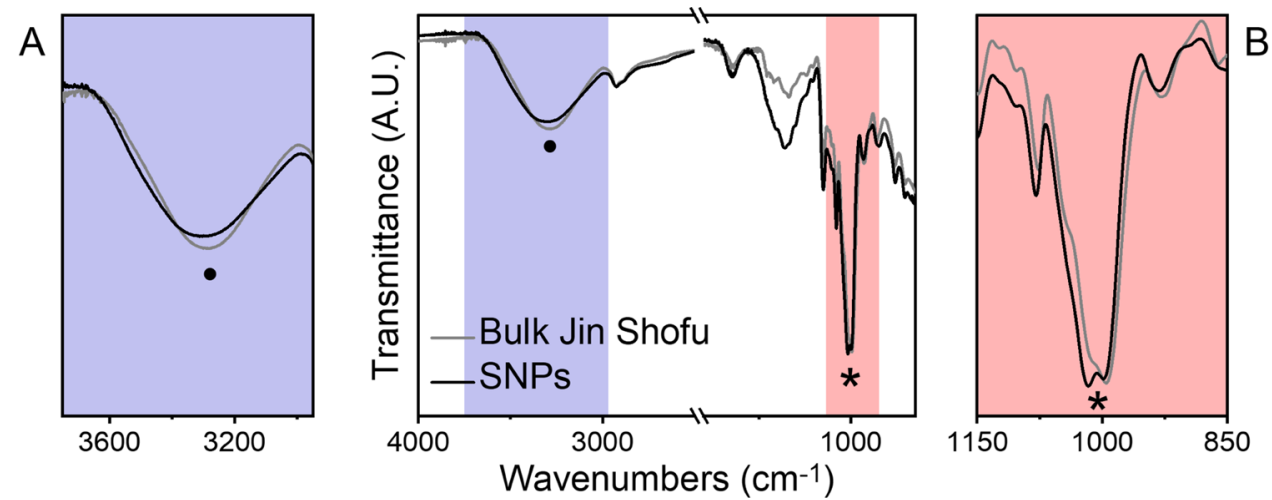

Figure 2. ATR-FTIR of bulk Jin Shofu (gray line) and Jin Shofu freeze-dried SNPs (black line). The insets detail the 3600-3300 (panel A, purpleshaded, left) and $1150-850 \mathrm{~cm}^{-1}$ (panel $\mathrm{B}$, pink-shaded, right) regions of the spectra.

minimizing the alkali content of the final SNPs formulation is crucial to avoid possible damages to the fragile paint layers. Treatment in $\mathrm{NaOH}$ under the same conditions left some nondissolved precipitate, possibly owing to the partial transformation of $\mathrm{NaOH}$ into carbonate. Extending heating time was discarded, as it resulted in strong yellowing of the starch dispersion; besides, precipitating such yellowed dispersions in ethanol led to the formation of macroscopic aggregates ( $\sim$ millimeter-sized flakes).

Two other fundamental parameters to optimize in the synthesis were the initial concentration of the bulk starch, prior to alkali dissolution, and the water/ethanol ratio used in the nonsolvent precipitation.

Tan et al. demonstrated that, when the initial starch concentration was in the $0.8-1 \%$ range $(w / v)$, precipitation in a nonsolvent of acetylated maize starch led to highly polydispersed SNPs, and increasing it up to $2 \%$ produced strong particle coagulation; these authors thus had to keep the concentration between 0.1 and $0.8 \%$ to achieve narrow size distributions and low polydispersity. The water/ethanol ratio used in the nonsolvent precipitation step, instead, is known to affect the morphology of the obtained SNPs. According to $\mathrm{Wu}$ et al., increasing the ratio in the 1:5-1:30 $(\mathrm{w} / \mathrm{w})$ range leads to a slight decrease in the SNPs mean size and smoother particle surfaces, while a change from elongated fibers to spheres was observed by Chin et al. when the ratio passes from 1:10 to $1: 20$. Pushing up to $1: 40$ was reported to yield small spherical SNPs $(\sim 25 \mathrm{~nm})$ mixed with larger fibers.

Starting from these considerations, we investigated the effect of the initial Jin Shofu starch concentration on the size distribution of the SNPs obtained following the precipitation step, varying also the water/ethanol ratio (see Figure 1). Because we aimed at a formulation for consolidating weak paint layers, potentially addressing large artifacts, we wanted to maximize the yield of the synthetic process, i.e., the final content of SNPs in the formulation. At the same time, small particle size $(<100 \mathrm{~nm})$, acceptable polydispersity, and spherical particle shape were desired. Besides, we wanted to keep the amount of nonaqueous solvent used in the synthesis as low as possible so as to have a green, affordable, and easily upscalable production route. Therefore, we had to look for the optimal balance between high initial concentration and low ethanol content.

We explored the $0.6-1.0 \% \mathrm{w} / \mathrm{v}$ starch concentration range, using a water/ethanol ratio of either $1: 10$ or $1: 40$. In all cases, our synthetic process has a yield $>97 \%$, which was deemed satisfactory for our applications. Interestingly, globular SNPs were obtained regardless of the initial starch concentration value and ethanol content (see Figure 1). The highest average particle size and polydispersity occur at $0.8 \% \mathrm{w} / \mathrm{v}$ concentration, at either $1: 10$ or $1: 40$. Particles of acceptable size $(<100 \mathrm{~nm})$ were obtained with the highest and lowest starch concentrations $(0.6$ and $1 \% \mathrm{w} / \mathrm{v})$; thus, we selected the combination of the highest concentration and the lowest ethanol content $(1 \% \mathrm{w} / \mathrm{v}$ and $1: 10$, respectively) as the precursor to the final particle dispersion.

The fact that, in all cases, we obtained spherical SNPs without the larger fiberlike structures observed by other authors at $1: 10$ and $1: 40$ solvent/nonsolvent ratios ${ }^{22,23}$ deserves some further considerations. The lower gelatinization temperature of wheat starch, as compared to other starch types, is likely key in facilitating the alkali disassembly and molecular weight reduction of the polymer chains. In turn, the presence of smaller and disassembled chains is expected to favor the reassembly of the polymer when exposed to the nonsolvent, by reducing steric hindrance. The absence of gluten (mainly composed of glutenin and gliadin) might also favor the process: while glutenin is defined as insoluble in most neutral solvents, gliadin has a solubility of $60-80 \%$ in alcohol; $^{24}$ we thus expected ethanol to be a more effective nonsolvent for gluten-removed starches such as Jin Shofu. These factors probably concur in promoting the formation of structures that minimize the interactions with ethanol (e.g., spheres) even at low nonsolvent contents. Because in our case the degradation of starch in alkali seems to be crucial for obtaining spherical SNPs of the desired size, we did not consider other synthetic approaches meant to avoid alteration of polymer molecular size, such as the use of hydrolysis protectors (urea $)^{25}$ or the aforementioned route that employs DMSO and autoclaves. ${ }^{21,25}$

The dry SNPs were analyzed by means of FTIR, XRD, and DSC, before their redispersion and application. The FTIR spectrum of the nanoparticles, compared with that of bulk Jin Shofu, is shown in Figure 2. Bulk starch typically has bands at $3000-2900 \mathrm{~cm}^{-1}$ (C-H stretching), $1150-1100 \mathrm{~cm}^{-1}$ (C-O, $\mathrm{C}-\mathrm{C}$, and $\mathrm{C}-\mathrm{O}-\mathrm{H}$ stretching), and $1100-900 \mathrm{~cm}^{-1}$ (C-O$\mathrm{H}$ bending). ${ }^{26}$ The spectrum of the SNPs shows a broader $\mathrm{OH}$ stretching band (figure FTIR, inset A) and inversion in the relative intensity of the peaks at 1015 and $995 \mathrm{~cm}^{-1}$ (figure FTIR, inset $B$ ). The $1050-950 \mathrm{~cm}^{-1}$ region is sensitive to the short-range order structures of the starch double helixes, and the relative intensity of bands around 1000, 1020, and 1050 


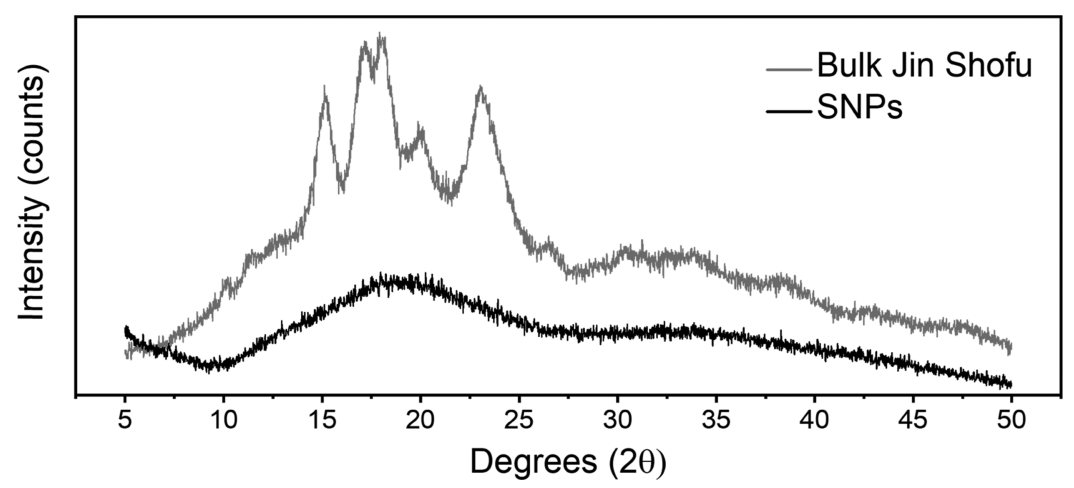

Figure 3. XRD of bulk Jin Shofu (gray line) and Jin Shofu freeze-dried SNPs (black line).
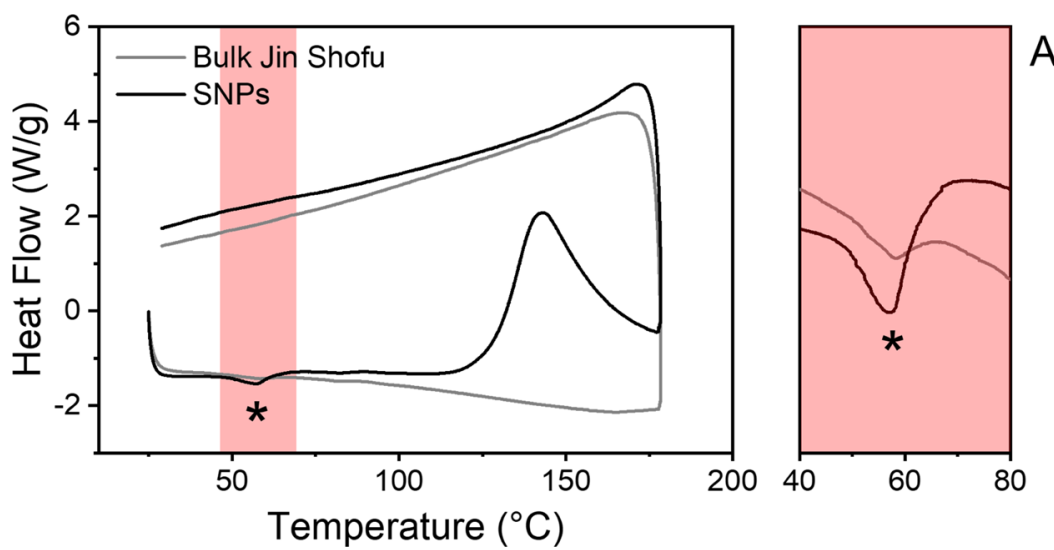

Figure 4. DSC thermograms of bulk Jin Shofu (gray line) and Jin Shofu freeze-dried SNPs (black line). Bottom curves were obtained during heating, and top curves were obtained during cooling. The pink-shaded inset (panel A) details the glass-transition temperatures in the $40-80^{\circ} \mathrm{C}$ interval.

$\mathrm{cm}^{-1}$ can be used as a marker for the presence of amorphous and ordered domains. ${ }^{27,28}$ Namely, the band at $1020 \mathrm{~cm}^{-1}$ increases in more amorphous samples, while those around 1000 and $1050 \mathrm{~cm}^{-1}$ become more defined in more crystalline samples. Indeed, the $1020 / 1000$ and $1050 / 1020$ band ratios have been used by some authors as measures of the short-range molecular order (Bello-Pérez et al., 2005; ${ }^{29}$ Brümmer et al., 2002; ${ }^{30}$ Iizuka and Aishima, $1999 ;^{31}$ Rubens et al., 1999; ${ }^{32}$ Smits et al., $\left.1998^{33}\right)$. In our case, the first ratio increases from $0.94 \pm 0.01$ to $1.02 \pm 0.02$, passing from bulk Jin Shofu to SNPs (normalizing the spectra at the maximum $\mathrm{CH}$ stretching absorption at $2930 \mathrm{~cm}^{-1}$ ), while the shoulder at $\mathrm{ca} 1045 \mathrm{~cm}^{-1}$ is observable only for bulk Jin Shofu.

XRD measurements are in good agreement with FTIR data: the spectrum of bulk Jin Shofu shows the typical diffraction peaks of type A crystalline starch at 15.1, 17.1, 18.0, 20.0, and $23.0^{\circ}$, with minor bands at $10.1,11.5$, and $26.5^{\circ} .^{28}$ Instead, the SNPs show only broad bands with no structure, clearly evidencing amorphousness, in agreement with other studies where alkalinization of starch is carried out (Figure 3). ${ }^{2,34}$

The thermograms of bulk Jin Shofu and SNPs are shown in Figure 4. As expected, the nanoparticles, being amorphous, exhibit a clear glass-transition event $\left(T_{\mathrm{g}} \sim 61{ }^{\circ} \mathrm{C}\right),{ }^{35}$ while bulk Jin Shofu has a weaker transition. The SNPs also show a large and pronounced exothermic peak centered at $143{ }^{\circ} \mathrm{C}$, which we attributed to structural rearrangement, ${ }^{36}$ as the chains gain mobility and relax the conformational stress acquired during fast assembly in the nonsolvent.
Overall, we can summarize the SNPs formation process as follows: bulk starch is largely disassembled and hydrolyzed during alkali treatment, possibly down to a short size; in turn, the presence of short and mobile chains, assisted by the absence of gluten, favors fast chain association during the nonsolvent precipitation step, promoting the formation of amorphous, rather than crystalline, particles.

Following the precipitation step and freeze-drying, the SNPs were redispersed in water. When the concentration is $10 \mathrm{~g} / \mathrm{L}$ or higher, the particles undergo rapid flocculation and sedimentation, to the point that their use would be practically discouraged. A concentration of either 1 or $5 \mathrm{~g} / \mathrm{L}$ was deemed as the lowest acceptable value for formulations meant to consolidate paint layers since repeated exposure to solvents through multiple treatments could be detrimental to sensitive artistic surfaces. At such concentration values, the spherical SNPs produce alkaline $\mathrm{pH}$ values $(8-10)$. Considering that the particles are thoroughly washed (and centrifuged) in ethanol before freeze-drying, the $\mathrm{pH}$ increase can be explained assuming that part of the $\mathrm{KOH}$ remains entrapped within the starch chains during the nonsolvent precipitation step, to be released when the polymer chains finally swell in water after redispersion. Capture of hydrophilic compounds by starch particles, followed by release in hydrophilic solvents, is indeed known in drug delivery. ${ }^{23}$ Highly alkaline environments can be harmful to pigments and oil binders in paint layers, e.g., through color alteration and oil saponification; ${ }^{37}$ however, dialysis of the SNPs dispersions overnight feasibly decreased the $\mathrm{pH}$ down to 6.5 , a value considered safe for the treatment 
of paintings. ${ }^{38}$ In principle, SNPs with some residual alkaline content could be useful for the treatment of acidic paper artworks, where concurrent readhesion of poorly bound dyes and deacidification of the cellulose substrate might be required.

Ethanol can also be used to blend with water for redispersing the SNPs. Even though its nonsolvent character toward starch promotes some aggregation of the particles, the presence of ethanol in the dispersing medium opens the application of SNPs onto water-sensitive paint layers, which are frequently found in art conservation. ${ }^{39}$ Besides, this solvent is a good choice as a particle carrier as it provides a good balance among surface wettability, volatility, and low toxicity. We monitored the dispersions' stability in the blends, systematically varying the relative alcohol content from 0 to $50 \%(\mathrm{v} / \mathrm{v}$, in steps of 5 ). The settling kinetics of the dispersions, evaluated by UV-vis spectroscopy, are shown in Figure 5. The aqueous dispersion

A

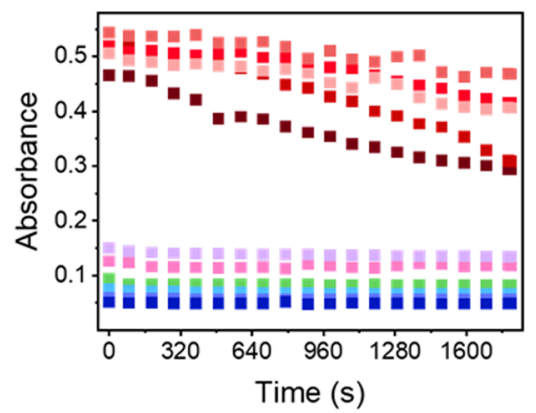

- $\mathrm{H}_{2} \mathrm{O}: \mathrm{EtOH} 50: 50$

- $\mathrm{H}_{2} \mathrm{O}: \mathrm{EtOH} 45: 55$

- $\mathrm{H}_{2} \mathrm{O}: \mathrm{EtOH} 40: 60$

- $\mathrm{H}_{2} \mathrm{O}$ :EtOH 35:65

$\mathrm{H}_{2} \mathrm{O}: \mathrm{EtOH} 30: 70$

$\mathrm{H}_{2} \mathrm{O}: \mathrm{EtOH} 25: 75$

$\mathrm{H}_{2} \mathrm{O}: \mathrm{EtOH} 80: 20$

$\mathrm{H}_{2} \mathrm{O}:$ EtOH 85:15

$\mathrm{H}_{2} \mathrm{O}: \mathrm{EtOH} 90: 10$

$\mathrm{H}_{2} \mathrm{O}: \mathrm{EtOH} 95: 5$

- $\mathrm{H}_{2} \mathrm{O}$ :EtOH 100:0

B

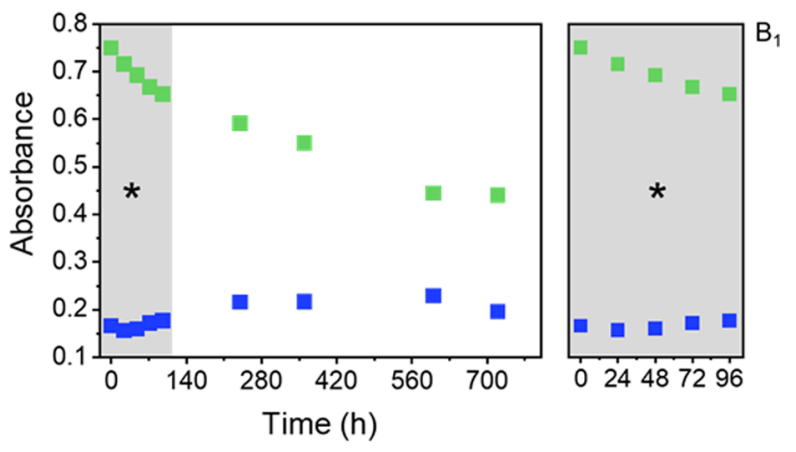

- SNPs $5 \mathrm{~g} / \mathrm{L}$ in $\mathrm{H}_{2} \mathrm{O}$ - SNPs $5 \mathrm{~g} / \mathrm{L}$ in $\mathrm{H}_{2} \mathrm{O}: \mathrm{EtOH} 85: 15$

Figure 5. Stability of Jin Shofu SNPs dispersions $(5 \mathrm{~g} / \mathrm{L})$ in water and water-ethanol blends at different ratios $(\mathrm{v} / \mathrm{v})$, measured by UV-vis spectroscopy. (A) Stability of dispersions with ethanol content from 0 to 50:50, over $30 \mathrm{~min}$ from preparation of the dispersions. (B) Stability of the water and $\mathrm{H}_{2} \mathrm{O}$ :EtOH 85:15 dispersions, over 1 month from preparation. The gray-shaded inset (panel $\mathrm{B}_{1}$ ) details their stability over the first $100 \mathrm{~h}$.

( $5 \mathrm{~g} / \mathrm{L}, 100 \%$ water) remains stable over 1 month, even though prolonged exposure to water produces the formation of aggregates visible to the naked eye, which remain suspended, likely due to the formation of microgel type of structures by the water-swollen starch particles. Among the hydroalcoholic dispersions $(5 \mathrm{~g} / \mathrm{L})$, those with ethanol content $>20 \%$ exhibit strong instability even within the first $30 \mathrm{~min}$ (see panel A in Figure 5). The higher absorbance, as compared to the aqueous system, is due to the partial aggregation of the particles promoted by the nonsolvent right after redispersion. The highest possible alcohol content to avoid fast particle sedimentation was $15 \%$, a system whose behavior is comparable to the aqueous dispersion within the first $24 \mathrm{~h}$ (panels $\mathrm{B}$ and $\mathrm{B}_{1}$ in Figure 5). Overall, only the water and $\mathrm{H}_{2} \mathrm{O}: \mathrm{EtOH} 85: 15(\mathrm{v} / \mathrm{v})$ systems, either at 1 or at $5 \mathrm{~g} / \mathrm{L}$, were considered suitable for the consolidation treatments and thus further characterized.

The hydrodynamic radius $\left(R_{\mathrm{h}}\right)$ values obtained by numberweighed DLS analysis (see Table 1 and Figure S1) confirm

Table 1. DLS and $\zeta$-Potential Data ${ }^{a}$

\begin{tabular}{|c|c|c|c|}
\hline sample & $R_{\mathrm{h}}$ intensity & $R_{\mathrm{h}} \underset{(\mathrm{nm})}{\text { number }}$ & $\zeta$ potential \\
\hline $\begin{array}{l}\text { SNPs } \mathrm{H}_{2} \mathrm{O} \text { dispersion } \\
1 \mathrm{~g} / \mathrm{L}\end{array}$ & $292.5 \pm 61.9$ & $47.0 \pm 15.5$ & $-0.9 \pm 1.4$ \\
\hline $\begin{array}{l}\text { SNPs } \mathrm{H}_{2} \mathrm{O}: \mathrm{EtOH} \\
85: 15(\mathrm{v} / \mathrm{v}) \text { dispersion } \\
1 \mathrm{~g} / \mathrm{L}\end{array}$ & $359.8 \pm 65.3$ & $44.9 \pm 28.9$ & $-3.2 \pm 2.6$ \\
\hline $\begin{array}{l}\text { SNPs } \mathrm{H}_{2} \mathrm{O} \text { dispersion } \\
5 \mathrm{~g} / \mathrm{L}\end{array}$ & $372.0 \pm 66.2$ & $32.2 \pm 18.1$ & $0.1 \pm 0.2$ \\
\hline $\begin{array}{l}\text { SNPs } \mathrm{H}_{2} \mathrm{O}: \text { EtOH } 85: 15 \\
(\mathrm{v} / \mathrm{v}) \text { dispersion } 5 \mathrm{~g} / \mathrm{L}\end{array}$ & $302.9 \pm 87.6$ & $239.7 \pm 42.4$ & $-1.9 \pm 1.0$ \\
\hline
\end{tabular}

${ }^{a}$ Hydrodynamic radius $\left(R_{\mathrm{h}}\right)$, obtained by intensity-weighted and number-weighted DLS, and $\zeta$ potential of Jin Shofu SNPs dispersions $(1$ and $5 \mathrm{~g} / \mathrm{L})$ in water and water-ethanol blend $(85: 15 \mathrm{v} / \mathrm{v})$.

that, shortly after preparation, dispersions at $1 \mathrm{~g} / \mathrm{L}$ have particle size of ca. $50 \mathrm{~nm}$ even when ethanol is added, comparable to the SNPs dimensions obtained right after nonsolvent precipitation. Raising the concentration up to $5 \mathrm{~g} /$ $\mathrm{L}$ produces a significant size increase for the hydroalcoholic dispersion, from $R_{\mathrm{h}} \sim 50$ to $\sim 250 \mathrm{~nm}$. The intensity-weighed $R_{\mathrm{h}}$ values are in the $300-370 \mathrm{~nm}$ range regardless of the dispersing medium and concentration ( 1 or $5 \mathrm{~g} / \mathrm{L}$ ); the higher dimensions as compared to the spherical particles observed with SEM (before redispersion) can be explained considering the fact that the swollen SNPs have high polydispersity. ${ }^{40}$ In all cases, the $\zeta$ potential of the SNPs is close to zero, as expected for uncharged polysaccharides like starch. ${ }^{41}$

Cryo-TEM images were collected on the $5 \mathrm{~g} / \mathrm{L}$ dispersion in water, while in the case of the hydroalcoholic dispersion, no clear images could be obtained, possibly due to the presence of ethanol that interferes with the sample freezing preparation. Figure 6 shows the presence of clusters of $\sim 50 \mathrm{~nm}$ that exhibit

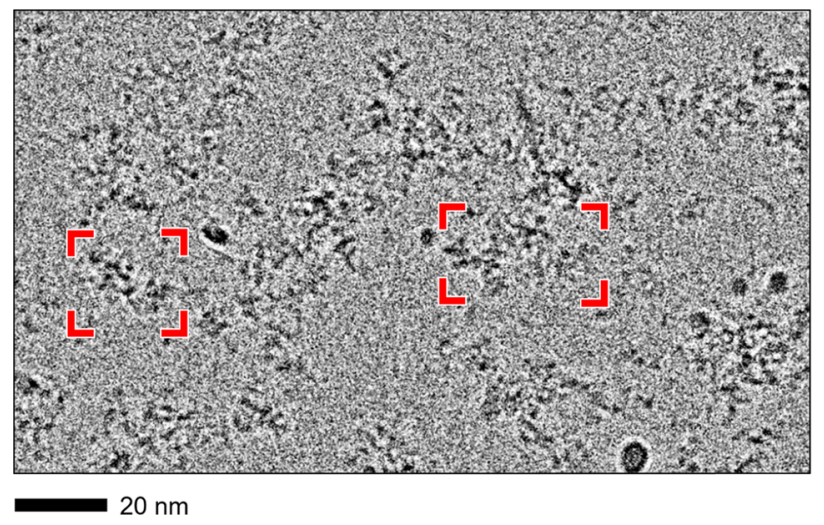

Figure 6. Cryo-TEM images of Jin Shofu SNPs dispersed in water (5 $\mathrm{g} / \mathrm{L}$ ). The red edges highlight two of the nanometric fractal gel-like clusters formed by nanosized starch units. The black circular spots are bubbles formed in the ice nanolayer. 


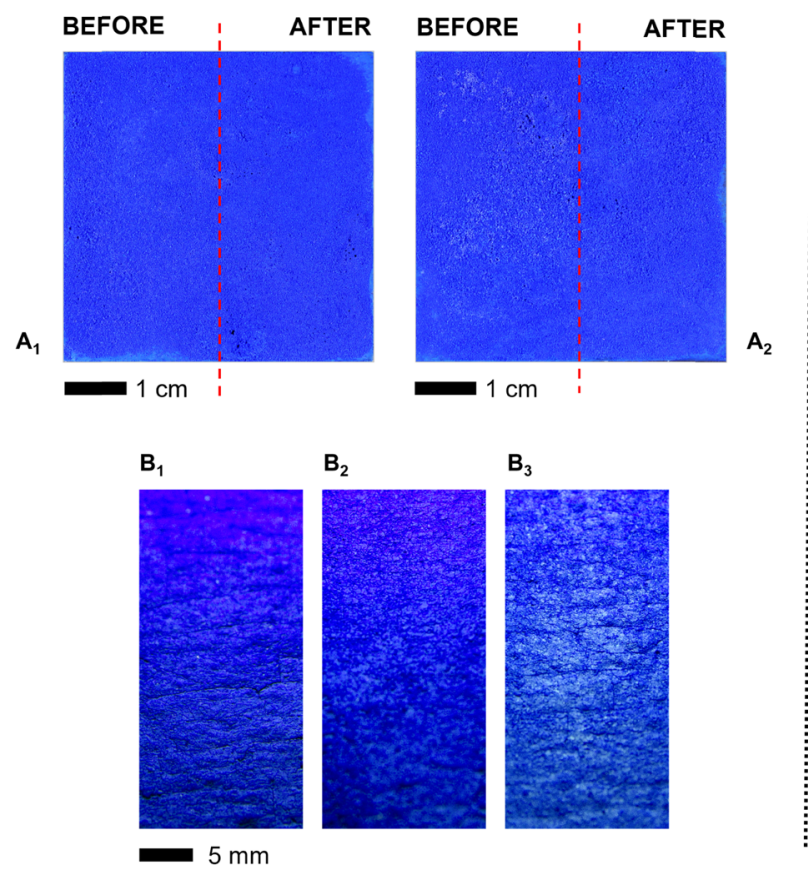

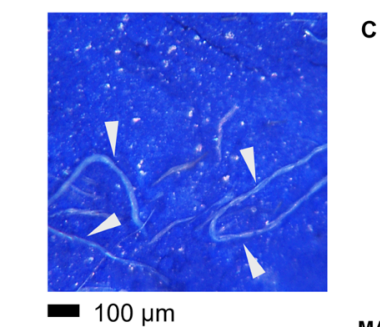

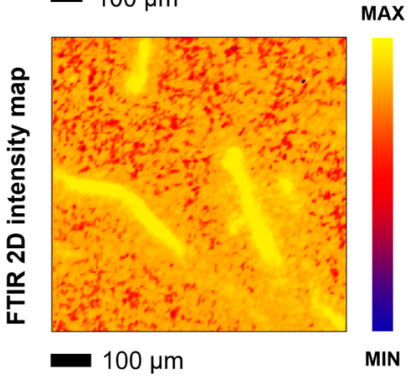

Figure 7. Ultramarine blue paint mock-ups before and after treatment with Jin Shofu starch (bulk or SNPs). In all panels, the mock-ups were treated by a single spray application with $1 \mathrm{~mL}$ of dispersion $(5 \mathrm{~g} / \mathrm{L})$. (A) Macrophotographs of mock-ups treated with the SNPs, using either water $\left(\mathrm{A}_{1}\right)$ or water-ethanol $\left(\mathrm{H}_{2} \mathrm{O}: \mathrm{EtOH} 85: 15 \mathrm{v} / \mathrm{v}, \mathrm{A}_{2}\right)$ dispersions. Half of the paint surface was left untreated as a reference. (B) Macrophotography comparison between mock-ups treated with the SNPs (dispersed in water, $\mathrm{B}_{1}$, or in the water-ethanol blend, $\mathrm{B}_{2}$ ) and with a dispersion of bulk Jin Shofu starch $\left(B_{3}\right)$; the latter forms a glossy superficial layer that alters the matte appearance of the paint. (C) Top: Microphotograph of the paint surface after treatment with the SNPs, showing microjunctions where the nanoparticles filled and consolidated cracks in the paint layer. Bottom: FTIR 2D imaging map of the $2990-2800 \mathrm{~cm}^{-1}$ IR spectral region, after subtraction of the ultramarine blue background spectra, acquired on the surface of the treated mock-ups; the orange-yellow pixels indicate strong adsorption by starch ( $\mathrm{CH}$ stretching bands), where the SNPs distribute on the surface and in the paint's cracks.

a fractal-like morphology produced by the organization of smaller units $(\sim 5 \mathrm{~nm})$. Similar gel-like fractals were observed by Putaux et al. for the structures formed by the aggregation of amylose or amylopectin following heat treatment and cooling. ${ }^{42}$ In particular, the primary amylose gelation mechanism is strictly related to conformational transitions from a random coil state to a gel-like structure with polymer-rich and deficient regions. Amylopectin aggregation exhibits similarities but with a looser organization owing to its branched structure. ${ }^{42}$ In our case, we conclude that the water-swollen SNPs can be structurally characterized as nanometric gel clusters.

Overall, we deemed the $5 \mathrm{~g} / \mathrm{L}$ SNPs dispersions, with either 0 or $15 \%$ ethanol content $(\mathrm{v} / \mathrm{v})$, as the best candidates for the applicative tests, based on the rationale of having the highest consolidant concentration and slightly decreased water content while keeping an acceptable particle size and dispersion stability.

Figure 7 shows the application of the dispersions to artificially aged painted mock-ups that mimic fragile, powdering painting surfaces. The SNPs were compared to a dispersion of bulk Jin Shofu starch at the same concentration. For each sample, half of the surface $\left(2.5 \times 5 \mathrm{~cm}^{2}\right)$ was treated by a single spray application with $1 \mathrm{~mL}$ of dispersion $(5 \mathrm{~g} / \mathrm{L})$, leaving the other half for monitoring possible color and surface alterations. Treatment with the SNPs did not alter the esthetic appearance of the ultramarine blue paint (Figure $7 \mathrm{~A}_{1}-\mathrm{A}_{2}$ ), and no color change was recorded as compared to the untreated surface $(\Delta E \sim 0.5)$. The nanoparticles distribute homogeneously on the surface (as observed by 2D FTIR imaging) and fill cracks in the degraded painted matrix, acting as micro- junctions (Figure 7C) that do not alter visually the pigment but provide reinforcement to the fragile paint. Only a slight change $(\Delta E \sim 2.0)$ was observed after three spraying rounds, which was still deemed acceptable (see Figure S2). Instead, the application of even a single spraying round with bulk starch produces the formation of a strongly glossy superficial starch layer that jeopardizes the matte finish of the pigment (Figure $\left.7 \mathrm{~B}_{3}\right)$ and causes some color alteration $(\Delta E \sim 2.5)$.

Figure 8 shows the distribution of SNPs and bulk starch across the section ( $1 \mathrm{~mm}$ thick) of treated paint mock-ups, as measured by $2 \mathrm{D}$ FTIR imaging. The presence of starch was monitored by mapping the intensity of the $\mathrm{CH}$ stretching band between 2990 and $2800 \mathrm{~cm}^{-1}$, after subtraction of the ultramarine blue background spectra. The untreated mock-up shows weak absorptions (reddish pixels on the blue background in the false color maps) due to impurities contained in the commercial pigment formulation. When a single round of spraying is used, the cross-section maps do not show significant absorption increase with respect to the untreated mock-up (results not shown), even though we cannot exclude that some SNPs distribute inside the matrix, but their presence in the FTIR maps is covered by the pigment's impurity absorptions. However, when three spraying rounds are applied (taking care to let the paint dry completely after each application), the penetration of the SNPs is evident: the particles cover completely the mock-up surface, distribute homogeneously across the section, and reach the paint bottom. Particles dispersed in water showed a slightly more complete distribution across the paint matrix compared to their water-ethanol analogue, which show some more accumulation 

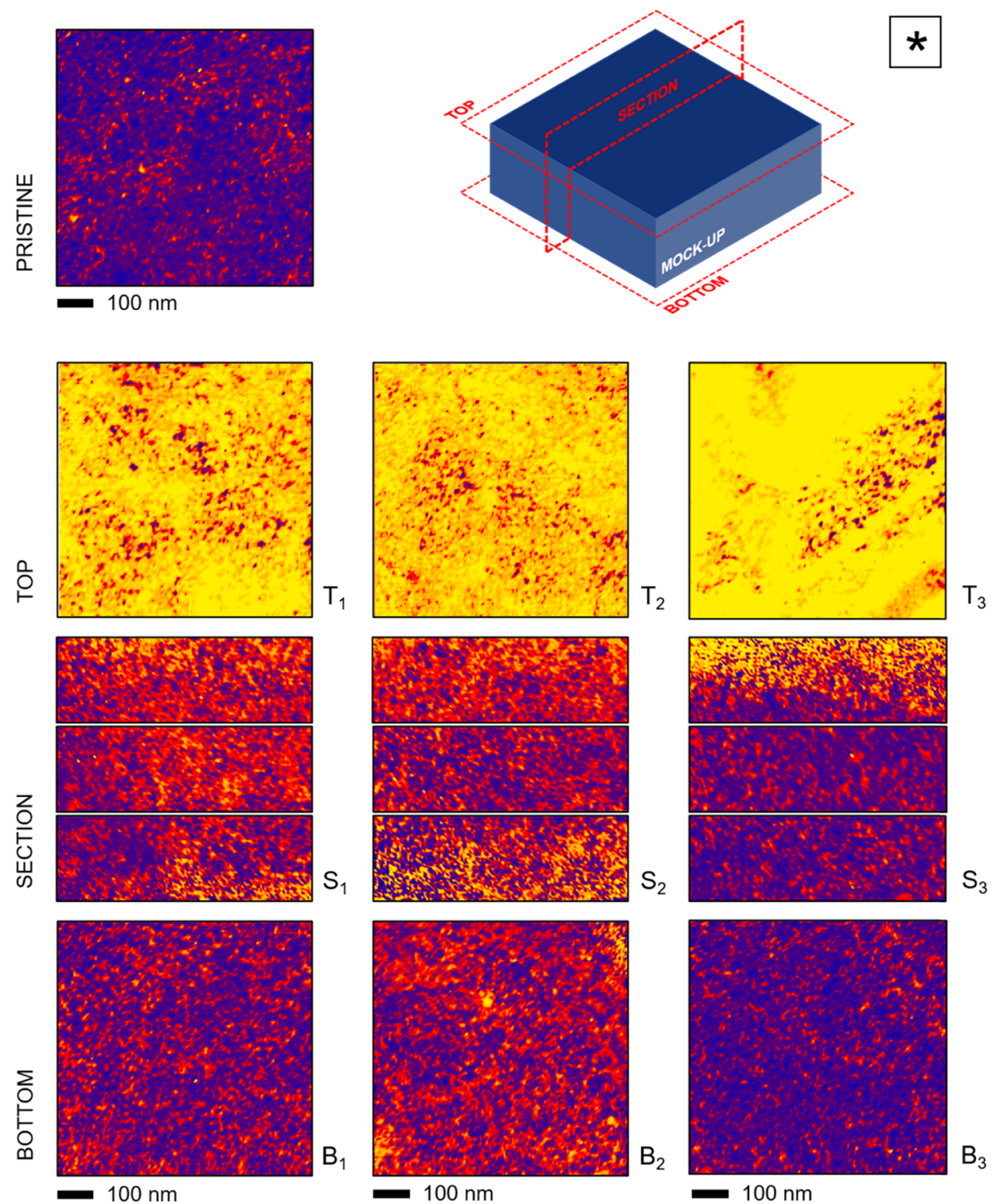

MIN

MAX

Figure 8. FTIR 2D imaging map of the $2990-2800 \mathrm{~cm}^{-1}$ IR spectral region, after subtraction of the ultramarine blue background spectra, acquired on a pristine paint mock-up (not treated with starch) and on aged mock-ups treated with Jin Shofu starch. Weak adsorptions in the map of the untreated mock-up are due to impurities contained in the commercial ultramarine blue formulation. Adsorption increases in the maps of the treated mock-ups are due to $\mathrm{CH}$ stretching bands of starch. For the treated mock-ups, maps were acquired on the top surface ("TOP" panels, $\mathrm{T}_{1-3}$ ), on the paints' cross section ("SECTION" panels, $\mathrm{S}_{1-3}$ ), and on the bottom surface ("BOTTOM" panels, $\mathrm{B}_{1-3}$ ); see also the mock-up scheme marked as “*”. For each row, subscripts indicate treatment with a dispersion of SNPs in water (1), water-ethanol blend $\left(\mathrm{H}_{2} \mathrm{O}: \mathrm{EtOH} 85: 15 \mathrm{v} / \mathrm{v}\right.$, (2), or with a dispersion of bulk Jin Shofu (3). The mock-ups were treated with three spray applications (each one with $1 \mathrm{~mL}$ of dispersion, $5 \mathrm{~g} / \mathrm{L}$ ).

in the bottom layers. Instead, bulk starch only accumulates on the surface, with no penetration inside the porous matrix. It must be noticed that, although accumulation in the top layers produces superficial consolidation, the presence of a strongly cohered surface film over poorly bound pigments is not desirable, as discontinuities in the mechanical properties across the section are known to favor cracking of paint layers. ${ }^{43}$

Figure 9 shows the amount of pigment removal from the mock-up surface by controlled contact with a fiberglass filter (1 $\mathrm{cm}^{2}$ ), before and after the application of the SNPs dispersions. We developed this semiquantitative in-house microsampling protocol (see Experimental Section) to overcome the limitations of traditional scotch tape and cotton swab tests. $^{44,45}$ Namely, scotch tape tests typically use strong adhesives to peel poorly adhered and cohered grains off the powdering surfaces; however, the forces involved in the peeling are too large and not representative of the actual mechanical stresses experienced by canvas paint layers. Rolling wet swabs on the surface provides only qualitative visual assessment of the pigments' cohesion, but it is not a reliable approach for gaining quantitative results. Instead, the fiberglass filter is able to give weak interactions with the ultramarine blue pigment grains, which is enough to compete with the scarce grain-grain cohesion forces and remove large portions of unbound 


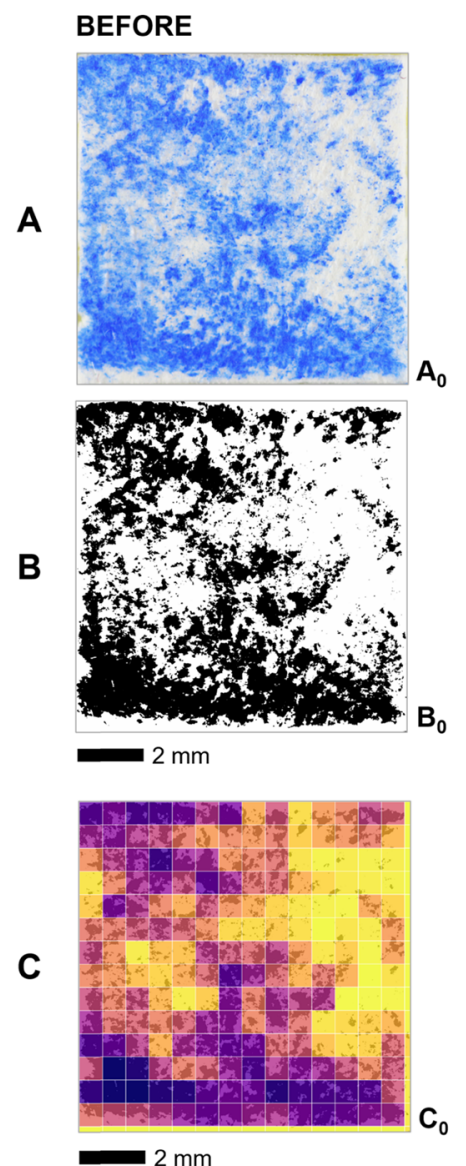

AFTER
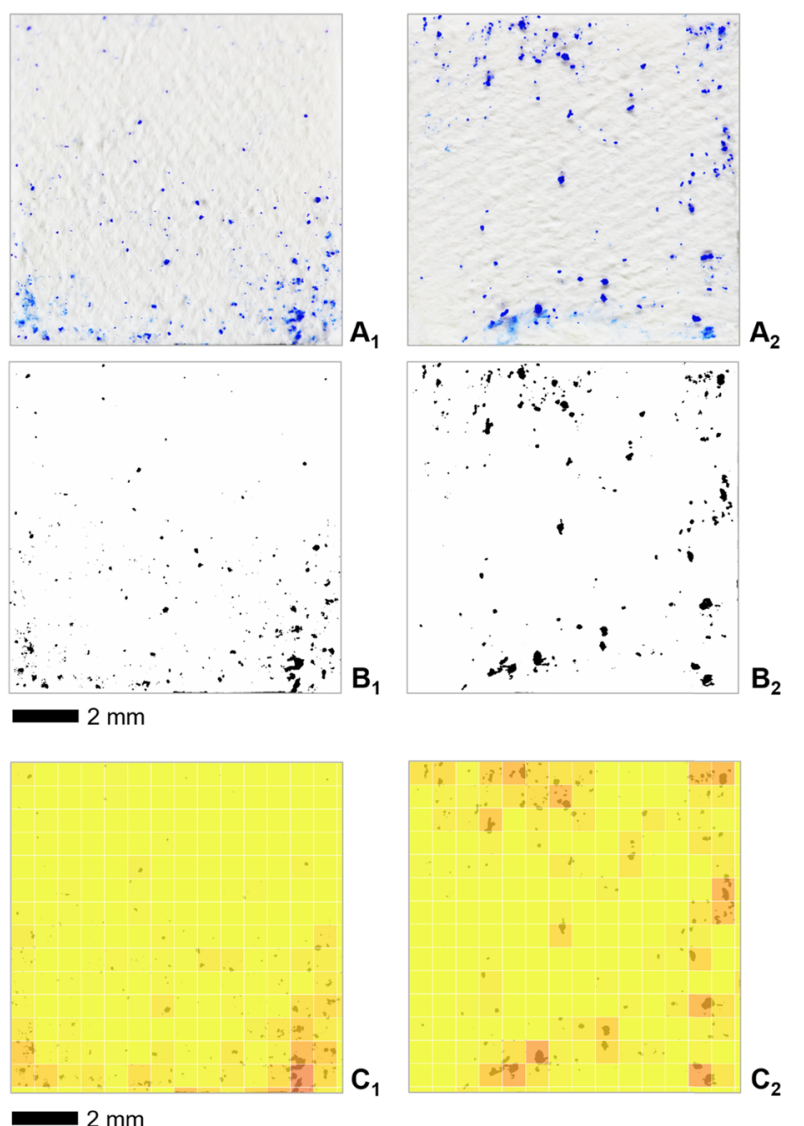

Pigment (relative coverage) \%

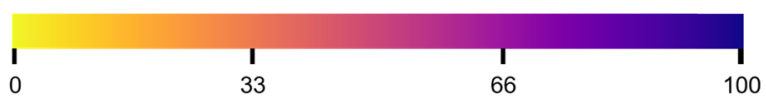

Figure 9. Macrophotographs of fiberglass filters $\left(1 \mathrm{~cm}^{2}\right)$ after contact with the ultramarine blue aged mock-ups. (A) Vis-light images; (B) binarized $\mathrm{b} / \mathrm{w}$ vis-light images; (C) binarized vis-light images in false colors; the surface is divided in tiles $\left(700 \times 700 \mu \mathrm{m}^{2}\right.$ each), and each tile is given a hue ranging from yellow ( $0 \%$ pigment coverage) to blue ( $100 \%$ coverage). For each row, the "0" subscript indicates that the filter was applied to the nonconsolidated aged paint, while " 1 " and " 2 " indicate application on paint surfaces consolidated with Jin Shofu SNPs dispersions (5 g/L) in water or water-ethanol $(85: 15 \mathrm{v} / \mathrm{v})$, respectively.

pigments without ripping the paint surface, as tape adhesives would. By controlling the weight applied on the filters (that have a standardized surface area) and the time of contact with the mock-up surface, we were able to obtain reproducible pigment removal.

Even by looking at the macrophotography images (Figure 9, panel series $\mathrm{A}_{0-2}$ and $\mathrm{B}_{0-2}$ ), it is evident that the mock-ups treated with either the water or water-ethanol SNPs dispersion lose minimal amounts of pigments as opposed to the untreated paint surface, where pigment removal by the filter is significantly high. To provide a better qualitative and semiquantitative evaluation, we divided the binarized images of the filter surface after contact with the pigment into tiles of 700 $\times 700 \mu \mathrm{m}^{2}$, and for each tile, the relative pigment coverage of the surface was calculated. Then, false color maps were obtained, where each tile is given a hue ranging from yellow ( $0 \%$ coverage) to blue $(100 \%$ coverage $)$; see Figure $9 \mathrm{C}_{0-2}$. When no consolidation treatment is carried out on the paint, the average pigment coverage of the filter is ca. $44 \%$, which is cut down to $1.5-3 \%$ when the paint layer is strengthened with the SNPs. This indicates that the starch particles effectively provided recohesion of the loose pigment grains, as both the water-ethanol and water dispersions proved highly beneficial; the latter is even more effective, in agreement with the more complete particle penetration observed through the paint cross sections (see above and Figure 8).

Pigment coverage of the filters' surfaces was also evaluated by FTIR 2D imaging, mapping the intensity of absorptions between 3715 and $3680 \mathrm{~cm}^{-1}$ (OH stretching bands of hydration water of silicates in the ultramarine blue pigment) on $700 \times 700 \mu \mathrm{m}^{2}$ tiles. The false color IR maps were then binarized, and the relative coverage was calculated (see Figure S3). The results are in good agreement with those obtained through macrophotography, as expected for a pigment like ultramarine blue, which absorbs strongly in both the vis and mid-IR spectra. While in this case having both the vis and IR light assessment is redundant, the possibility of carrying out two valid and complementary tests is useful in cases where the pigment has poor vis-light contrast with the filter (white colors) or is mixed with transparent components that absorb strongly in the IR (e.g., oil binders). 


\section{CONCLUSIONS}

We synthesized Jin Shofu starch nanoparticles (SNPs) through an optimized bottom-up approach based on alkali dissolution and nonsolvent precipitation and applied the SNPs for the first time for the consolidation of fragile, poorly bound paint layers. Our synthetic approach is robust and reliable (yield $\geq 97 \%$ ), does not use additives potentially harmful to the paints, and can be tuned to obtain globular particles with different size distributions. Simply varying the length of the dialysis step allows tuning the $\mathrm{pH}$ of the dispersions down to harmless values (6.5), i.e., the residual $\mathrm{KOH}$ included in the particles is easily removed before application of the dispersions.

The low gelatinization temperature of wheat starch is likely key in promoting alkali disassembly and molecular weight reduction of the polymer chains, lowering steric hindrance and thus favoring the formation of spherical and compact particles through exposure to the nonsolvent (ethanol). The absence of ethanol-soluble gliadin in the gluten-removed Jin Shofu starch probably further promotes the process. Fast reassembly in ethanol leads to highly amorphous particles, as verified by $\mathrm{XRD}$, FTIR, and thermal analyses. In principle, amorphous starch domains with high chain mobility might favor mechanical stress relaxation and polymer-pigment interactions when the particles are applied to fragile paint layers. Besides, they favor optical transparency as opposed to crystalline polymer centers found in bulk starch.

Overall, this synthetic route for the formulation of starch consolidants proved to be a promising, less expensive, and "greener" alternative to acid hydrolysis or starch dissolution in DMSO. Once precipitated, the SNPs can be redispersed in water or water-ethanol blends, where maximum stability is exhibited for ethanol content $\leq 15 \%(\mathrm{v} / \mathrm{v})$. Cryo-TEM showed that, upon swelling in the aqueous phase, the particles form fractal nanometric gel clusters $(\sim 50 \mathrm{~nm})$ produced by the organization of nanosized units in polymer-rich and -poor domains.

Once applied to fragile, matte ultramarine blue paint layers, the Jin Shofu SNPs were able to penetrate through the paint matrix, distributing through its cross section without inducing esthetic alterations. The nanoparticles also fill cracks in the paint, acting like microjunctions. Instead, dispersions of bulk starch accumulate on the paint surface, forming glossy superficial films that jeopardize the matte finish of the pigment and alter its color. The SNPs provide significant strengthening to the weak, poorly bound paints. The consolidation effect was evaluated using an in-house methodology specifically developed for fragile painted layers: pigment removal, measured semiquantitatively through standardized microsampling followed by microphotography and FTIR $2 \mathrm{D}$ imaging, is cut down to minimal values when the paints are consolidated, while it takes place significantly for the untreated pigments. A single spray application (in our case, $1 \mathrm{~mL}$ for ca. $12 \mathrm{~cm}^{2}, 5 \mathrm{~g} /$ $\mathrm{L}$ in water or $\mathrm{H}_{2} \mathrm{O}: \mathrm{EtOH} 85: 15 \mathrm{v} / \mathrm{v}$ ) was sufficient to provide effective consolidation; however, multiple applications might also be carried out (e.g., up to 3), depending on the conservation status of the artifact.

In conclusion, our synthesis and application of Jin Shofu SNPs open up new perspectives in the conservation of fragile paint layers, where effective and safe consolidants are still sorely needed.

Future upgrades might involve the surface functionalization of the SNPs to further improve their stability in nonaqueous solvents and their interaction with pigments, as the presence of -OH groups and the particles' high specific area offer good potentiality for surface chemical modification. ${ }^{9}$ The application of the SNPs dispersions to real degraded paintings will also have to be assessed. In this case, we might expect the SNPs to interact also with binders, as amylose is capable of forming complexes with lipids and proteins (Bhatnagar and Hanna, 1994; ${ }^{46}$ Biliaderis and Seneviratne, $1990 ;{ }^{47}$ Copeland et al., $2009{ }^{48}$ Godet, Bizot, and Buleon, $1995^{49}$ ). Finally, the Jin Shofu SNPs might also be useful as controlled releasers of hydrophilic compounds either in cultural heritage preservation (release of antifungal or anticorrosion agents) or in drugdelivery processes.

\section{ASSOCIATED CONTENT}

\section{Supporting Information}

The Supporting Information is available free of charge at https://pubs.acs.org/doi/10.1021/acsami.1c11064.

DLS plots of Jin Shofu SNPs dispersions ( 1 and $5 \mathrm{~g} / \mathrm{L}$ ) in water and water-ethanol blend $(85: 15 \mathrm{v} / \mathrm{v})$; macrophotographs of ultramarine blue paint mock-ups before and after treatment with Jin Shofu starch (bulk or SNPs) by three spray applications; and comparison of image analysis results between macrophotographs and FTIR 2D imaging of fiberglass filters after contact with the ultramarine blue aged mock-ups (PDF)

\section{AUTHOR INFORMATION}

\section{Corresponding Authors}

David Chelazzi - Department of Chemistry "Ugo Schiff" and CSGI, University of Florence, I-50019 Florence, Italy; ○ orcid.org/0000-0001-9994-3356;

Email: david.chelazzi@unifi.it

Rodorico Giorgi - Department of Chemistry "Ugo Schiff" and CSGI, University of Florence, I-50019 Florence, Italy; (1) orcid.org/0000-0002-7752-4107;

Email: rodorico.giorgi@unifi.it

\section{Author}

Andrea Casini - Department of Chemistry "Ugo Schiff" and CSGI, University of Florence, I-50019 Florence, Italy; (1) orcid.org/0000-0003-4256-8887

Complete contact information is available at: https://pubs.acs.org/10.1021/acsami.1c11064

\section{Author Contributions}

R.G. and A.C. conceived the idea of this study; A.C. and D.C. designed and conducted the experiments and analyzed the experimental data; R.G. and D.C. supervised the experiments; and A.C. and D.C. wrote the manuscript. All authors have given approval to the final version of the manuscript.

\section{Funding}

A.C., D.C., and R.G. received funding from MUR (PRIN 2017249YEF), CSGI (CMPT191138 MIUR), and the University of Florence Department of Chemistry (center of excellence).

\section{Notes}

The authors declare no competing financial interest.

\section{ACKNOWLEDGMENTS}

MUR (PRIN 2017249YEF), CSGI, and the University of Florence Department of Chemistry (center of excellence) are 
acknowledged for funding this work. Antonio Mirabile (paper conservator) is gratefully acknowledged for providing the Jin Shofu starch and for his useful comments and discussion. Annalisa Guerri and Giancarlo Tria (Flocem, University of Florence, Department of Chemistry) are kindly acknowledged for assistance on the Cryo-TEM measurements. Irina Crina Anca Sandu (Munch Museum Conservation Department) is gratefully acknowledged for useful discussion.

\section{REFERENCES}

(1) Pinna, D.; Galeotti, M.; Mazzeo, R. Scientific Examination for the Investigation of Paintings: A Handbook for Conservator-Restorers; Centro Di, 2009.

(2) Hansen, E. F.; Walston, S.; Bishop, M. H. Matte Paint: Its History and Technology, Analysis, Properties and Conservation Treatment, With a Special Emphasis on Ethnographic Objects; Getty Conservation Institute; United Kingdom Institute for Conservation of Historic and Artistic Works, 1994.

(3) Gohde Sandbakken, E.; Storevik Tveit, E. Preserving a Master: Edvard Munch and His Painted Sketches. J. Urban Cult. Res. 2012, 5, 86-104.

(4) Frøysaker, T.; Streeton, N. L. W.; Kutzke, H.; Hanssen-Bauer, F.; Topalova-Casadiego, B. Public Paintings by Edvard Munch and His Contemporaries: Change and Conservation Challenges; Archetype Publications: London, 2015.

(5) Chelazzi, D.; Chevalier, A.; Pizzorusso, G.; Giorgi, R.; Menu, M.; Baglioni, P. Characterization and Degradation of Poly(Vinyl Acetate)Based Adhesives for Canvas Paintings. Polym. Degrad. Stab. 2014, 107, 314-320.

(6) Chelazzi, D.; Badillo-Sanchez, D.; Giorgi, R.; Cincinelli, A.; Baglioni, P. Self-Regenerated Silk Fibroin with Controlled Crystallinity for the Reinforcement of Silk. J. Colloid Interface Sci. 2020, 576, $230-240$.

(7) Alexopoulou, I.; Zervos, S. Paper Conservation Methods: An International Survey. J. Cult. Heritage 2016, 21, 922-930.

(8) Yu, L.; Liu, X.; Petinakis, E.; Dean, K.; Bateman, S. Starch Based Blends, Composites and Nanocomposites. In Advances in Natural Polymers; Springer: Berlin, 2013; pp 121-154.

(9) le Corre, D.; Bras, J.; Dufresne, A. Starch Nanoparticles: A Review. Biomacromolecules 2010, 11, 1139-1153.

(10) Morán, D.; Gutiérrez, G.; Blanco-López, M. C.; Marefati, A.; Rayner, M.; Matos, M. Synthesis of Starch Nanoparticles and Their Applications for Bioactive Compound Encapsulation. Appl. Sci. 2021, $11,4547$.

(11) Mittal, V. Nanocomposites with Biodegradable Polymers: Synthesis, Properties, and Future Perspectives; Oxford University Press, 2011.

(12) Tanio, N.; Koike, Y. What Is the Most Transparent Polymer? Polym. J. 2000, 32, 43-50.

(13) Rivas, C. J. M.; Tarhini, M.; Badri, W.; Miladi, K.; GreigeGerges, H.; Nazari, Q. A.; Galindo Rodríguez, S. A.; Román, R. A.; Fessi, H.; Elaissari, A. Nanoprecipitation Process: From Encapsulation to Drug Delivery. Int. J. Pharm. 2017, 532, 66-81.

(14) Rueden, C. T.; Schindelin, J.; Hiner, M. C.; DeZonia, B. E.; Walter, A. E.; Arena, E. T.; Eliceiri, K. W. ImageJ2: ImageJ for the next Generation of Scientific Image Data. BMC Bioinf. 2017, 18, No. 529.

(15) Jivan, M. J.; Madadlou, A.; Yarmand, M. An Attempt to Cast Light into Starch Nanocrystals Preparation and Cross-Linking. Food Chem. 2013, 141, 1661-1666.

(16) Hunt, R. W. G.; Pointer, M. R. Measuring Colour (The WileyIS\&T Series in Imaging Science and Technology); Wiley, 2011.

(17) Badillo-Sanchez, D.; Chelazzi, D.; Giorgi, R.; Cincinelli, A.; Baglioni, P. Characterization of the Secondary Structure of Degummed Bombyx Mori Silk in Modern and Historical Samples. Polym. Degrad. Stab. 2018, 157, 53-62.

(18) Mastrangelo, R.; Chelazzi, D.; Poggi, G.; Fratini, E.; Pensabene Buemi, L.; Petruzzellis, M. L.; Baglioni, P. Twin-Chain Polymer
Hydrogels Based on Poly(Vinyl Alcohol) as New Advanced Tool for the Cleaning of Modern and Contemporary Art. Proc. Natl. Acad. Sci. USA 2020, 117, 7011-7020.

(19) Rueden, C. T.; Schindelin, J.; Hiner, M. C.; DeZonia, B. E.; Walter, A. E.; Arena, E. T.; Eliceiri, K. W. ImageJ2: ImageJ for the next Generation of Scientific Image Data. BMC Bioinf. 2017, 18, No. 529.

(20) Chinga, G.; Syverud, K. Quantification of Paper Mass Distributions within Local Picking Areas. Nord. Pulp Pap. Res. J. 2007, 22, 441-446.

(21) Saari, H.; Fuentes, C.; Sjöö, M.; Rayner, M.; Wahlgren, M. Production of Starch Nanoparticles by Dissolution and Non-Solvent Precipitation for Use in Food-Grade Pickering Emulsions. Carbohydr. Polym. 2017, 157, 558-566.

(22) Chin, S. F.; Pang, S. C.; Tay, S. H. Size Controlled Synthesis of Starch Nanoparticles by a Simple Nanoprecipitation Method. Carbohydr. Polym. 2011, 86, 1817-1819.

(23) Gutiérrez, G.; Morán, D.; Marefati, A.; Purhagen, J.; Rayner, M.; Matos, M. Synthesis of Controlled Size Starch Nanoparticles (SNPs). Carbohydr. Polym. 2020, 250, No. 116938.

(24) Cook, W. H.; Rose, R. C. Solubility of Gluten. Nature 1934, 380-381.

(25) Perez-Rea, D.; Bergenståhl, B.; Nilsson, L. Development and Evaluation of Methods for Starch Dissolution Using Asymmetrical Flow Field-Flow Fractionation. Part I: Dissolution of Amylopectin. Anal. Bioanal. Chem. 2015, 407, 4315-4326.

(26) Cael, J. J.; Koenig, J. L.; Blackwell, J. Infrared and Raman Spectroscopy of Carbohydrates. Part VI: Normal Coordinate Analysis of V-amylose. Biopolymers 1975, 14, 1885-1903.

(27) Warren, F. J.; Gidley, M. J.; Flanagan, B. M. Infrared Spectroscopy as a Tool to Characterise Starch Ordered Structure A Joint FTIR-ATR, NMR, XRD and DSC Study. Carbohydr. Polym. 2016, 139, 35-42.

(28) Pozo, C.; Rodríguez-Llamazares, S.; Bouza, R.; Barral, L.; Castaño, J.; Müller, N.; Restrepo, I. Study of the Structural Order of Native Starch Granules Using Combined FTIR and XRD Analysis. J. Polym. Res. 2018, 25, No. 266.

(29) Bello-Perez, L. A.; De Francisco, A.; Agama-Acevedo, E.; Gutierrez-Meraz, F.; Garcia-Suarez, F. J.L. Morphological and Molecular Studies of Banana Starch. Food Sci. Technol. Int. 2005, 11 (5), 367-372.

(30) Brummer, T.; Meuser, F.; van Lengerich, B.; Niemann, C. Effect of Extrusion Cooking on Molecular Parameters of Corn Starch. Starch/Stärke 2002, 54 (1), 1-8.

(31) Iizuka, K.; Aishima, T. Starch Gelation Process Observed by FT-IR/ATR Spectrometry with Multivariate Data Analysis. J. Food Sci. 1999, 64 (4), 653-658.

(32) Rubens, P; Snauwaert, J; Heremans, K; Stute, R In situ observation of pressure-induced gelation of starches studied with FTIR in the diamond anvil cell. Carbohydr. Polym. 1999, 39 (3), 231235.

(33) Smits, A. L. M.; Ruhnau, F. C.; Vliegenthart, J. F. G.; van Soest, J. J. G. Ageing of Starch Based Systems as Observed with FT-IR and Solid State NMR Spectroscopy. Starch/Stärke 1998, 50 (11-12), 478-483.

(34) Ahmad, M.; Gani, A.; Hassan, I.; Huang, Q.; Shabbir, H. Production and Characterization of Starch Nanoparticles by Mild Alkali Hydrolysis and Ultra-Sonication Process. Sci. Rep. 2020, 10, No. 3533.

(35) Zeleznak, K. J.; Hoseney, R. C. The Glass Transition in Starch. Cereal Chem. 1987, 64, 121-124.

(36) Razavi, M.; Zhang, W.; Khonakdar, H. A.; Janke, A.; Li, L.; Wang, S. Q. Inducing Nano-Confined Crystallization in PLLA and PET by Elastic Melt Stretching. Soft Matter 2021, 17, 1457-1462.

(37) Byrne, A. Wolbers Cleaning Methods: Introduction; AICCM Bulletin: Routledge, 1991; pp 3-11.

(38) Ormsby, B.; Lee, J.; Bonaduce, I.; Lluveras-Tenorio, A. Evaluating Cleaning Systems for Use on Water Sensitive Modern 
Oil Paints: A Comparative Study. In Conservation of Modern Oil Paintings; Springer International Publishing, 2019; pp 11-35.

(39) Mills, L.; Jan van den Berg, K.; Burnstock, A.; de Groot, S.; Megens, L.; Bisschoff, M.; van Keulen, H.; Duarte, F. Water Sensitivity of Modern Artists' Oil Paints. Preprints of the 15th Triennial Meeting of the International Council of Museums Conservation Committee in Delhi 2008, 2, 651-659.

(40) Hassan, P. A.; Rana, S.; Verma, G. Making Sense of Brownian Motion: Colloid Characterization by Dynamic Light Scattering. Langmuir 2015, 31, 3-12.

(41) Salemis, P.; Rinaudo, M. Gel Permeation Chromatography of Starch and Other Uncharged Polysaccharides. Polym. Bull. 1984, 11, 397-400.

(42) Putaux, J. L.; Buléon, A.; Chanzy, H. Network Formation in Dilute Amylose and Amylopectin Studied by TEM. Macromolecules 2000, 33, 6416-6422.

(43) Pauchard, L.; Giorgiutti-Dauphiné, F. Craquelures and Pictorial Matter. J. Cult. Heritage. 2020361-373. DOI: 10.1016/ j.culher.2020.08.007.

(44) Drdácký, M.; Lesák, J.; Rescic, S.; Sližková, Z.; Tiano, P.; Valach, J. Standardization of Peeling Tests for Assessing the Cohesion and Consolidation Characteristics of Historic Stone Surfaces. Mater. Struct. 2012, 45, 505-520.

(45) Baglioni, P.; Vargas, R. C.; Chelazzi, D.; Gonzàlez, M. C.; Desprat, A.; Giorgi, R. The Maya Site Of Calakmul: In Situ Preservation Of Wall Paintings And Limestone Using Nanotechnology. Stud. Conserv. 2006, 51, 162-169.

(46) Bhatnagar, S.; Milford, A. H. Amylose-Lipid Complex Formation During Single-Screw Extrusion of Various Corn Starches. Cereal Chem. 1994, 71 (6), 582-587.

(47) Seneviratne, H. D.; Biliaderis, C. D. Action of $\alpha$-Amylases on Amylose-Lipid Complex Superstructures. J. Cereal Sci. 1991, 13 (2), 129-143.

(48) Copeland, L.; Blazek, J.; Salman, H.; Tang, M. C. Form and Functionality of Starch. Food Hydrocolloids 2009, 23 (6), 1527-1534.

(49) Godet, M. C.; Bizot, H.; Buléon, A. Crystallization of AmyloseFatty Acid Complexes Prepared with Different Amylose Chain Lengths. Carbohydr. Polym. 1995, 27 (1), 47-52. 